Pacific Journal of Mathematics

THE INDETERMINATE RATE PROBLEM FOR BIRTH-DEATH 


\title{
THE INDETERMINATE RATE PROBLEM FOR BIRTH-DEATH PROCESSES
}

\author{
ERIK A. VAN DOORN
}

\begin{abstract}
A birth-death process is completely determined by its set of rates if and only if this set satisfies a certain condition $C$, say. If for a set of rates $R$ the condition $C$ is not fulfilled, then the problem arises of characterizing all birth-death processes which have rate set $R$ (the indeterminate rate problem associated with $R$ ). We show that the characterization may be effected by means of the decay parameter, and we determine the set of possible values for the decay parameter in terms of $R$. A fundamental role in our analysis is played by a duality concept for rate sets, which, if the pertinent rate sets satisfy $C$, obviously leads to a duality concept for birth-death processes. The latter can be stated in a form which suggests the possibility of extension in the context of indeterminate rate problems. This, however, is shown to be only partially true.
\end{abstract}

1. Introduction. Let $\mathscr{P}=\left\{p_{i j}(t) \mid i, j \in E^{\prime}, 0 \leq t<\infty\right\}$ be the set of transition probability functions of a standard, time-homogeneous Markov process $X(t)$ on the state space $E^{\prime} \equiv\{-1,0,1, \ldots\}$. That is,

$$
p_{i j}(t) \equiv \operatorname{Pr}\{X(s+t)=j \mid X(s)=i\}
$$

and

$$
\begin{gathered}
p_{i j}(t) \geq 0, \\
\sum_{k \in E^{\prime}} p_{i k}(t) \leq 1, \\
\sum_{k \in E^{\prime}} p_{i k}(s) p_{k j}(t)=p_{i j}(s+t), \\
\lim _{t \downarrow 0} p_{i j}(t)=p_{i j}(0)=\delta_{i j},
\end{gathered}
$$

$\left(i, j \in E^{\prime} ; s, t \geq 0\right) . \mathscr{P}$ is said to represent a birth-death process if the $Q$-matrix $Q=\left(q_{i j}\right)$ of $\mathscr{P}$, defined by

$$
q_{i j} \equiv \lim _{t \downarrow 0} t^{-1}\left(p_{i j}(t)-\delta_{i j}\right),
$$

satisfies $q_{l j}=0$ if $i=-1$ or $|i-j|>1,0<q_{\imath j}<\infty$ if $i \geq 0$ and $|i-j|$ $=1$ (with the exception $\left.0 \leq q_{0,-1}<\infty\right)$, and

$$
q_{l i}=-\left(q_{l, i-1}+q_{i, i+1}\right), \quad i \geq 0 .
$$


It then follows that the functions $p_{\imath j}(t)$ satisfy the backward equations

$$
p_{i j}^{\prime}(t)=\sum_{k \in E^{\prime}} q_{i k} p_{k j}(t) \text {. }
$$

In addition the transition probabilities of a birth-death process are always supposed to fulfill the forward equations

$$
p_{i j}^{\prime}(t)=\sum_{k \in E^{\prime}} p_{i k}(t) q_{k j} .
$$

Clearly, with this definition of a birth-death process, the state -1 is absorbing and the set $E \equiv\{0,1,2, \ldots\}$ constitutes an irreducible class of states. Consequently [15], for $i, j \in E$,

$$
\gamma(\mathscr{P}) \equiv-\lim _{t \rightarrow \infty} t^{-1} \log p_{i j}(t)
$$

exists, is non-negative, and is independent of $i$ and $j$. We shall adhere to Kingman's [15] terminology by referring to $\gamma(\mathscr{P})$ as the decay parameter of $\mathscr{P}$. We remark that in some papers [2], [23], however, the concept of decay parameter is defined in terms of the functions $p_{i j}(t)-p_{J}$, where

$$
p_{J} \equiv \lim _{t \rightarrow \infty} p_{i j}(t)
$$

instead of the probabilities $p_{\imath j}(t)$. Evidently, both interpretations are identical if $p_{j}=0$ for all $j \in E$, that is, if the process is transient or null-recurrent.

We write

$$
\lambda_{l}(\mathscr{P}) \equiv q_{i, i+1}, \quad \mu_{l}(\mathscr{P}) \equiv q_{i, l-1}
$$

$(i \in E)$ and call these quantities the birth rate and death rate, respectively, of $\mathscr{P}$ in state $i . R(\mathscr{P}) \equiv\left\{\lambda_{i}(\mathscr{P}), \mu_{i}(\mathscr{P})\right\}_{i=0}^{\infty}$ is the set of birth and death rates of $\mathscr{P}$. More generally, any set of real numbers $R \equiv\left\{\lambda_{i}, \mu_{i}\right\}_{l=0}^{\infty}$, where $\lambda_{i}, \mu_{i+1}>0(i \geq 0)$ and $\mu_{0} \geq 0$, will be called a set of birth and death rates (or a rate set, for short), since for any such set $R$ there is at least one birth-death process $\mathscr{P}$ such that $R(\mathscr{P})=R([\mathbf{1 0}],[11],[17])$. Several authors [11], [12], [14] have shown that the rate set $\left\{\lambda_{i}, \mu_{i}\right\}$ uniquely determines a birth-death process if and only if the series

$$
\sum_{n=0}^{\infty}\left\{\pi_{n}+\left(\lambda_{n} \pi_{n}\right)^{-1}\right\}
$$

diverges, where

$$
\pi_{0} \equiv 1, \quad \pi_{n} \equiv \frac{\lambda_{0} \lambda_{1} \cdots \lambda_{n-1}}{\mu_{1} \mu_{2} \cdots \mu_{n}} \quad(n>0) .
$$


In this paper we will focus on the problem of characterizing all birth-death processes having the same rate set $R \equiv\left\{\lambda_{i}, \mu_{i}\right\}$ for which the series (1.8) converges. This problem will be referred to as the indeterminate rate problem (IRP) associated with $R$.

It is known [11], [12] that an IRP admits of a one-parameter family of solutions. In Feller's [11] analysis the characteristic parameter $\alpha$ is interpreted in terms of the solution it identifies by means of a "boundary condition at infinity"; the set of possible values for $\alpha$ is shown to be the interval $[0, \infty]$, independent of the specific IRP at hand. In Karlin and McGregor's [12] approach the characteristic parameter $\xi$, say, is identified with the smallest point in the support of a certain measure on the nonnegative real axis which is always associated with a birth-death process; the precise set of possible values for $\xi$, which now depends on the specific rate set at hand, is determined only in the case $\mu_{0}=0$.

We shall show that one has a more appealing option in that the decay parameter of a birth-death process can serve as a characteristic parameter if the process is not uniquely determined by its rates. Actually, it is not difficult to see that the decay parameter can be identified with Karlin and McGregor's characteristic parameter, so that the problem remains of determining the precise set of possible values for the decay parameter of a solution of an IRP in the case $\mu_{0}>0$. All this will be elaborated in $\S 4$, while $\$ \S 2$ and 3 contain the necessary preliminary results.

A birth-death process represented by $\left\{p_{i j}(t)\right\}$ will be called honest if equality prevails in (1.2) for all $i \in E^{\prime}$ and $t \geq 0$. Reuter [16] has shown that an IRP has exactly one honest solution. In $\$ 5$ we shall identify the honest solution of an IRP.

In the course of our analysis we make use of a duality concept for rate sets which links the sets where $\mu_{0}=0$ with those where $\mu_{0}>0$. If each of a pair of dual rate sets uniquely determines a birth-death process, then this duality concept for rate sets implies a duality concept for birth-death processes, which, in fact, is equivalent to that introduced by Siegmund [19] in a more general context. In $\S 6$ we will discuss the possibility of extending this duality concept for birth-death processes beyond the bounds of dual rate sets which uniquely determine the associated processes.

2. Preliminaries. For a rate set $R \equiv\left\{\lambda_{i}, \mu_{i}\right\}$ we define the polynomials $Q_{n}(x) \equiv Q_{n}(x ; R)$ by the recurrence formula

$$
\begin{aligned}
\lambda_{n} Q_{n+1}(x) & =\left(\lambda_{n}+\mu_{n}-x\right) Q_{n}(x)-\mu_{n} Q_{n-1}(x), \quad n>0, \\
Q_{0}(x) & =1, \quad \lambda_{0} Q_{1}(x)=\lambda_{0}+\mu_{0}-x
\end{aligned}
$$


We shall have reference to the simple result

$$
Q_{n}(0)=1+\mu_{0} \sum_{k=0}^{n-1}\left(\lambda_{k} \pi_{k}\right)^{-1},
$$

with $\pi_{k}$ being defined in (1.9). To facilitate comparison with the relevant literature, we also observe that the monic polynomials

$$
P_{n}(x)=(-1)^{n} \lambda_{0} \lambda_{1} \cdots \lambda_{n-1} Q_{n}(x), \quad n>0,
$$

satisfy the recurrence

$$
\begin{gathered}
P_{n+1}(x)=\left(x-\lambda_{n}-\mu_{n}\right) P_{n}(x)-\lambda_{n-1} \mu_{n} P_{n-1}(x), \quad n>0, \\
P_{0}(x)=1, \quad P_{1}(x)=x-\lambda_{0}-\mu_{0} .
\end{gathered}
$$

From [5] it now follows that for all positive $n$ the polynomial $Q_{n}(x)$ has $n$ real, simple zeros

$$
x_{n 1}(R)<x_{n 2}(R)<\cdots<x_{n n}(R) .
$$

These zeros have the property

$$
0<x_{n+1, i}(R)<x_{n i}(R)<x_{n+1, i+1}(R)
$$

$(i=1,2, \ldots, n ; n=1,2, \ldots)$, whence

$$
\xi_{i}(R) \equiv \lim _{n \rightarrow \infty} x_{n l}(R)
$$

$(i=1,2, \ldots)$ exists, and

$$
0 \leq \xi_{i}(R) \leq \xi_{i+1}(R)<\infty .
$$

Furthermore,

$$
\xi_{i+1}(R)=\xi_{i}(R) \Rightarrow \xi_{n}(R)=\xi_{l}(R) \text { for all } n>i .
$$

Throughout the remainder of this section we assume that $R$ defines an IRP, that is, the series (1.8) converges. From, essentially, Stieltjes [20] (cf. also [1], [4], [24]), we then have

$$
\sum_{i=1}^{\infty}\left\{\xi_{i}(R)\right\}^{-1}<\infty
$$

implying in particular

$$
0<\xi_{i}(R)<\xi_{l+1}(R) \text { and } \xi_{l}(R) \rightarrow \infty \quad(i \rightarrow \infty),
$$

by virtue of (2.6) and (2.7). Moreover,

$$
Q_{\infty}(x) \equiv \lim _{n \rightarrow \infty} Q_{n}(x)
$$


exists, and is an entire function whose set of zeros equals the unbounded set of positive numbers

$$
\Xi(R) \equiv\left\{\xi_{1}(R), \xi_{2}(R), \ldots\right\}
$$

With the sequence $\left\{Q_{n}\right\}$, and hence with $R$, we can associate a Stieltjes moment problem (SMP) by asking for a positive measure $d \psi$ on the nonnegative real axis with respect to which the polynomials $Q_{n}(x)$ are orthogonal. This SMP is solvable [5], [12] and we can normalize the measure such that

$$
\int_{0}^{\infty} Q_{i}(x) Q_{j}(x) d \psi(x)=\pi_{i}^{-1} \delta_{\imath j}
$$

( $i, j \in E$; in particular $\int_{0}^{\infty} d \psi(x)=1$ ). Moreover, since $R$ defines an IRP, the SMP is indeterminate, that is, there are infinitely many solutions [12, Thms. 14 and 15]. We shall be interested in the extremal solutions, which may be defined as follows [18].

Let

$$
\rho(x) \equiv\left\{\sum_{n=0}^{\infty} \pi_{n} Q_{n}^{2}(x)\right\}^{-1},
$$

then $\rho(x)$ is positive for all real $x$ and equals, if $x \geq 0$, the maximal mass any solution of the SMP can concentrate at $x$. Supposing that a solution of the SMP locates positive mass at the point $x$; then that solution is an extremal solution if and only if the point mass at $x$ equals $\rho(x)$.

Some pertinent properties of extremal solutions are the following [18]. There is an one-to-one correspondence between the real numbers in the interval $\left[0, \xi_{1}(R)\right]$ and the extremal solutions of the indeterminate SMP associated with $R$. For $\xi \in\left[0, \xi_{1}(R)\right]$ we denote the corresponding extremal solution by $d \psi(\cdot ; \xi)$. The support of $d \psi(\cdot ; \xi)$ is discrete and consists of the point $\xi$ and exactly one point in each of the intervals $\left(\xi_{i}(R), \xi_{l+1}(R)\right], i=1,2, \ldots$; the support of $d \psi\left(\cdot ; \xi_{1}(R)\right)$ equals $\Xi(R)$. Finally, the supporting points of two different extremal solutions strictly separate each other.

We will have use for the next lemma concerning the moments of order -1 of the extremal solutions of the SMP associated with $R$. For convenience we write

$$
\phi(\xi) \equiv \int_{0}^{\infty} x^{-1} d \psi(x ; \xi)
$$

and note that $\phi(\xi)$ exists for $0<\xi \leq \xi_{1}(R)$.

LEMMA 1. As $\xi$ increases from 0 to $\xi_{1}(R)$, the function $\phi(\xi)$ decreases continuously from $+\infty$ to some positive value which, if $\mu_{0}>0$, is strictly less than $\mu_{0}^{-1}$. 
Proof. From [12, pp. 530-531] we know that $\phi(\xi)$ is continuous on the interval $\left(0, \xi_{1}(R)\right]$, and that $\mu_{0} \phi\left(\xi_{1}(R)\right)<1$. Chihara [6, pp. 338-340] shows that an extremal solution of the indeterminate SMP associated with $R$ is uniquely determined by its moment of order -1 , the latter ranging between some positive number and infinity. (Actually, in [6], as in [18], extremal solutions are indexed by their moments of order -1 with reversed sign.) It follows that $\phi(\xi)$ must be strictly decreasing on the interval $\left(0, \xi_{1}(R)\right]$.

3. Duality for rate sets. The following duality concept for rate sets was introduced in [13] (see also [2], [21], [23]). Let $R \equiv\left\{\lambda_{i}, \mu_{l}\right\}$ be a rate set then the dual set $R^{d}=\left\{\lambda_{i}^{d}, \mu_{i}^{d}\right\}$ is defined by

$$
\begin{aligned}
& \mu_{0}=0 \Rightarrow \lambda_{l}^{d}=\mu_{l+1}, \quad \mu_{l}^{d}=\lambda_{l}, \\
& \mu_{0}>0 \Rightarrow \lambda_{l}^{d}=\mu_{l}, \quad \mu_{0}^{d}=0, \quad \mu_{i+1}^{d}=\lambda_{i}
\end{aligned}
$$

$(i=0,1, \ldots)$. Clearly, this duality concept establishes a one-to-one correspondence between the rate sets where $\mu_{0}=0$ and those where $\mu_{0}>0$. As regards the parameters defined in (1.9) we evidently have

$$
\begin{aligned}
& \mu_{0}=0 \Rightarrow \pi_{n}^{d}=\lambda_{0}\left(\lambda_{n} \pi_{n}\right)^{-1} \\
& \mu_{0}>0 \Rightarrow \pi_{n}^{d}=\mu_{0}\left(\mu_{n} \pi_{n}\right)^{-1}
\end{aligned}
$$

$(n=0,1, \ldots)$, where $\pi_{n}\left(\pi_{n}^{d}\right)$ corresponds to $R\left(R^{d}\right)$. Since $\lambda_{n} \pi_{n}=$ $\mu_{n+1} \pi_{n+1}$, it follows that

$$
\sum_{n=0}^{\infty}\left\{\pi_{n}+\left(\lambda_{n} \pi_{n}\right)^{-1}\right\}<\infty \Leftrightarrow \sum_{n=0}^{\infty}\left\{\pi_{n}^{d}+\left(\lambda_{n}^{d} \pi_{n}^{d}\right)^{-1}\right\}<\infty,
$$

that is, $R$ defines an IRP if and only if $R^{d}$ defines an IRP.

In the remainder of this section we shall assume that $R \equiv\left\{\lambda_{i}, \mu_{l}\right\}$ defines an IRP, whence also the SMP associated with $R$ is indeterminate. Explicit reference to $R$ will be suppressed, thus we write $\xi_{i}$ for $\xi_{i}(R)$. Further, it will be convenient to assume $\mu_{0}=0$. All quantities pertaining to the rate set $R^{d}$ will be indicated by an asterisk. In particular, $\mu_{0}^{*} \equiv \mu_{0}^{d}$ $>0, Q_{n}^{*}(x) \equiv Q_{n}\left(x ; R^{d}\right)$ and $\xi_{i}^{*} \equiv \xi_{l}\left(R^{d}\right)$; also, $d \psi^{*}(\cdot ; \xi), 0 \leq \xi \leq \xi_{1}^{*}$, denotes the extremal solution of the (indeterminate) SMP associated with $R^{d}$ of which $\xi$ is the smallest supporting point.

It is now easy to see that the polynomials $Q_{n}^{*}(x)$ are precisely the kernel polynomials with parameter 0 corresponding to $\left\{Q_{n}(x)\right\}[5$, p. 37 and Thm. I.9.1]. Actually, one has [23]

$$
Q_{n}^{*}(x)=\lambda_{n} \pi_{n}\left(Q_{n+1}(x)-Q_{n}(x)\right) /(-x) .
$$


From (2.1) and (3.4) one easily obtains

$$
\sum_{k=0}^{n} \pi_{k} Q_{k}^{2}(x)=\lambda_{0}^{-1} x \sum_{k=0}^{n} \pi_{k}^{*}\left(Q_{k}^{*}(x)\right)^{2}+Q_{n+1}(x) Q_{n}^{*}(x),
$$

which, by letting $n$ tend to infinity and in view of (2.11), leads to

$$
\{\rho(x)\}^{-1}=x\left\{\lambda_{0} \rho^{*}(x)\right\}^{-1}+Q_{\infty}(x) Q_{\infty}^{*}(x) .
$$

As regards the zeros of $Q_{\infty}(x)$ and $Q_{\infty}^{*}(x)$, there is the separation result [6], [12]

$$
0<\xi_{i}<\xi_{l}^{*}<\xi_{l+1}, \quad i=1,2, \ldots
$$

Now let $d \psi$ denote any solution of the SMP associated with $R$. By [12, Lemma 3] it then follows that

$$
d \theta(x) \equiv \lambda_{0}^{-1} x d \psi(x), \quad x \geq 0,
$$

is a solution of the SMP associated with $R^{d}$. It will be of interest to us to ascertain whether or not the measure defined by (3.8) is extremal when $d \psi$ is extremal. The answer is given in the next lemma.

LEMMA 2. (i) If $d \psi(\cdot)=d \psi(\cdot ; 0)$, then $d \theta(\cdot)=d \psi^{*}\left(\cdot ; \xi_{1}^{*}\right)$;

(ii) If $d \psi(\cdot)=d \psi\left(\cdot ; \xi_{1}\right)$, then $d \theta(\cdot)=d \psi^{*}\left(\cdot ; \xi_{1}\right)$;

(iii) If $d \psi(\cdot)=d \psi(\cdot ; \xi)$, where $0<\xi<\xi_{1}$, then $d \theta(\cdot)$ is not extremal.

Proof. (i) Let $\xi$ denote the first positive point in the support of $d \psi(\cdot ; 0)$. Then, since 0 is the first point in the support of $d \psi(\cdot ; 0)$, $0<\xi_{1}<\xi<\xi_{2}$. The mass concentrated by $d \psi(\cdot ; 0)$ at the point $\xi$ equals $\rho(\xi)$, for $d \psi(\cdot ; 0)$ is extremal. Consequently,

$$
d \theta(\xi)=\left\{\left(\rho^{*}(\xi)\right)^{-1}+\lambda_{0} \xi^{-1} Q_{\infty}(\xi) Q_{\infty}^{*}(\xi)\right\}^{-1} .
$$

It is evident from (2.2) that $Q_{\infty}(x)>0$ if $x<\xi_{1}$, so, since $\xi$ lies between the first two zeros of $Q_{\infty}(x)$, we must have $Q_{\infty}(\xi)<0$. Further, since $d \theta$ is a solution of the SMP for $R^{d}$, we have, by [5, Thm. II.4.4 (i)], $\xi \leq \xi_{1}^{*}$, whence $Q_{\infty}^{*}(\xi) \geq 0$. From (3.9) it now follows that $d \theta(\xi) \geq \rho^{*}(\xi)$. But since $\rho^{*}(\xi)$ is the maximal mass any solution of the SMP for $R^{d}$ can have at $\xi$, we must have $Q_{\infty}^{*}(\xi)=0$ and $d \theta(\xi)=\rho^{*}(\xi)$. Consequently, $\xi=\xi_{1}^{*}$ and $d \theta$ is extremal, whence $d \theta(\cdot)=d \psi^{*}\left(\cdot ; \xi_{1}^{*}\right)$.

(ii) Since $Q_{\infty}\left(\xi_{1}\right)=0$, we see from (3.6) and (3.8) that the mass concentrated at $\xi_{1}$ by $d \theta$ is precisely $\rho^{*}\left(\xi_{1}\right)$, whence $d \theta$ is extremal. Considering (3.7) it follows that $d \theta(\cdot)=d \psi^{*}\left(\cdot ; \xi_{1}\right)$. 
(iii) If $0<\xi<\xi_{1}$ and $d \psi(\cdot)=d \psi(\cdot ; \xi)$, then $d \theta$ concentrates mass at the point $\xi$, while $Q_{\infty}(\xi) Q_{\infty}^{*}(\xi)>0$. The latter, together with (3.9) implies $d \theta(\xi)<\rho^{*}(\xi)$, so that $d \theta$ is not extremal.

Of prime importance in the next section will be the observation that, by Lemma 2(ii) (recall that $\mu_{0}^{*}=\lambda_{0}$ ),

$$
\mu_{0}^{*} \int_{0}^{\infty} x^{-1} d \psi^{*}\left(x ; \xi_{1}\right)=\int_{0}^{\infty} d \psi\left(x ; \xi_{1}\right)=1 .
$$

So, $\xi_{1}$ is the (only) point in the interval $\left(0, \xi_{1}^{*}\right]$, where the function $\phi(\xi)$ of (2.12) and Lemma 1, defined in terms of $R^{d}$, has the value $\left(\mu_{0}^{*}\right)^{-1}$.

We finally note from Lemma 2 that the extremal solutions $d \psi(\cdot ; 0)$ and $d \psi\left(\cdot ; \xi_{1}\right)$ of the SMP associated with $R$ can be expressed in terms of the extremal solutions $d \psi^{*}\left(\cdot ; \xi_{1}\right)$ and $d \psi^{*}\left(\cdot ; \xi_{1}^{*}\right)$, respectively, of the SMP associated with $R^{d}$ as

$$
d \psi(x ; 0)= \begin{cases}1-\mu_{0}^{*} \int_{0}^{\infty} y^{-1} d \psi^{*}\left(y ; \xi_{1}^{*}\right), & x=0 \\ \mu_{0}^{*} x^{-1} d \psi^{*}\left(x ; \xi_{1}^{*}\right), & x>0\end{cases}
$$

and

$$
d \psi\left(x ; \xi_{1}\right)= \begin{cases}0, & x=0 \\ \mu_{0}^{*} x^{-1} d \psi^{*}\left(x ; \xi_{1}\right), & x>0 .\end{cases}
$$

Using (3.6) it may be shown that a similar representation for the solutions $d \psi(\cdot ; \xi)$, where $0<\xi<\xi_{1}$, is not possible.

4. Characterizing the solutions of an IRP. We return to the context of $\S 1$ and let $\mathscr{P}=\left\{p_{i j}(t)\right\}$ represent a birth-death process whose rate set $R \equiv R(\mathscr{P})$ defines an IRP. Karlin and McGregor [12, Thm. 12] then show that there is a unique extremal solution $d \psi(\cdot ; \xi(\mathscr{P}))$ of the indeterminate SMP associated with $R$ such that

$$
p_{i j}(t)=\pi_{j} \int_{0}^{\infty} e^{-x t} Q_{i}(x) Q_{j}(x) d \psi(x ; \xi(\mathscr{P}))
$$

$(i, j \in E ; t \geq 0)$, where $0 \leq \xi(\mathscr{P}) \leq \xi_{1}(R)$ and the parameters $\pi_{n}$ and the polynomials $Q_{n}$ are defined in terms of $R$ as in (1.9) and (2.1). As for the transition probabilities involving the state -1 , we note that by (1.4) and the forward equations (1.6)

$$
p_{i,-1}(t)=\mu_{0} \int_{0}^{t} p_{i 0}(\tau) d \tau
$$


( $i \in E ; t \geq 0$ ), while $p_{-1, j}(t)=\delta_{-1, j}$ for all $j \in E^{\prime}$ and $t \geq 0$ by (1.4) and the backward equations (1.5).

From the representation formula (4.1) we observe the following

LeMMA 3. The decay parameter of $\mathscr{P}$ equals the first point in the support of the extremal solution of the SMP for $R$ corresponding to $\mathscr{P}$.

Proof. Taking $i=j=0$ in (4.1) one has

$$
p_{00}(t)=\int_{0}^{\infty} e^{-x t} d \psi(x ; \xi(\mathscr{P}))=e^{-\xi(\mathscr{P}) t}\left\{a+\mathcal{O}\left(e^{-b t}\right)\right\}
$$

as $t \rightarrow \infty$, where $a=d \psi(\xi(\mathscr{P}) ; \xi(\mathscr{P}))>0$ and $b>\xi_{1}(R)-\xi(\mathscr{P}) \geq 0$. Hence,

$$
\gamma(\mathscr{P})=-\lim _{t \rightarrow \infty} t^{-1} \log p_{00}(t)=\xi(\mathscr{P})
$$

This result proves our statement in the Introduction that a birth-death process is uniquely determined by its rate set and its decay parameter. The next result gives the range of possible values for $\gamma(\mathscr{P})$, given that the rate set $R \equiv R(\mathscr{P})$ defines an IRP.

THEOREM 1. (i) If $\mu_{0}(\mathscr{P})=0$, then $0 \leq \gamma(\mathscr{P}) \leq \xi_{1}(R)$;

(ii) If $\mu_{0}(\mathscr{P})>0$, then $\xi_{1}\left(R^{d}\right) \leq \gamma(\mathscr{P}) \leq \xi_{1}(R)$.

Proof. In view of Lemma 3, the first statement as well as the upper bound in the second statement follow from Karlin and McGregor's representation theorem. As for the lower bound in (ii), we note from (1.2), (4.1) and (4.2) that for all $t \geq 0$

$$
1 \geq p_{0,-1}(t)=\mu_{0}(\mathscr{P}) \int_{0}^{t} \int_{0}^{\infty} e^{-x \tau} d \psi(x ; \xi(\mathscr{P})) d \tau .
$$

The assumption $\xi(\mathscr{P})=0$ obviously leads to a contradiction, so we have $\xi(\mathscr{P})>0$. Then, by interchanging the integrals,

$$
\begin{aligned}
p_{0,-1}(t)= & \mu_{0}(\mathscr{P}) \int_{0}^{\infty} x^{-1} d \psi(x ; \xi(\mathscr{P})) \\
& -\mu_{0}(\mathscr{P}) \int_{0}^{\infty} x^{-1} e^{-x t} d \psi(x ; \xi(\mathscr{P})) .
\end{aligned}
$$

Letting $t$ tend to infinity, it follows that

$$
\mu_{0}(\mathscr{P}) \int_{0}^{\infty} x^{-1} d \psi(x ; \xi(\mathscr{P})) \leq 1,
$$


whence, by virtue of Lemmas 1 and 3 and (3.10),

$$
\gamma(\mathscr{P})=\xi(\mathscr{P}) \geq \xi_{1}\left(R^{d}\right) .
$$

We will now show that every possible value for $\gamma(\mathscr{P})$ indicated in Theorem 1 can in fact occur.

THEOREM 2. Let $R \equiv\left\{\lambda_{i}, \mu_{i}\right\}$ be a rate set defining an IRP and let $\gamma \in\left[0, \xi_{1}(R)\right]$ if $\mu_{0}=0$ and $\gamma \in\left[\xi_{1}\left(R^{d}\right), \xi_{1}(R)\right]$ if $\mu_{0}>0$. Then the functions $p_{i j}(t ; \gamma), i, j \in E^{\prime}, t \geq 0$, defined by

$$
p_{i j}(t ; \gamma) \equiv \pi_{j} \int_{0}^{\infty} e^{-x t} Q_{i}(x) Q_{j}(x) d \psi(x ; \gamma)
$$

$(i, j \in E)$, together with $p_{l,-1}(t ; \gamma) \equiv \mu_{0} \int_{0}^{t} p_{t 0}(\tau ; \gamma) d \tau \quad(i \in E)$ and $p_{-1, j}(t ; \gamma) \equiv \delta_{-1, j}\left(j \in E^{\prime}\right)$, are the transition probability functions of $a$ birth-death process with rate set $R$ and decay parameter $\gamma$.

Proof. The result is mainly due to Karlin and McGregor [12, Thms. $2,4,5$ and 9]. The only thing we have to prove is that (1.2) is valid for $\left\{p_{i j}(t: \gamma)\right\}$. This we do by strengthening [12, Thms. 7]. Indeed, if $\mu_{0}>0$, we have

$$
\begin{aligned}
p_{i,-1}(t ; \gamma) & =\mu_{0} \int_{0}^{t} p_{i 0}(\tau ; \gamma) d \tau=\mu_{0} \int_{0}^{t} \int_{0}^{\infty} e^{-x t} Q_{i}(x) d \psi(x ; \gamma) d \tau \\
& =\mu_{0} \int_{0}^{\infty} x^{-1} Q_{\imath}(x) d \psi(x ; \gamma)-\mu_{0} \int_{0}^{\infty} x^{-1} e^{-x t} Q_{\imath}(x) d \psi(x ; \gamma) .
\end{aligned}
$$

Adding this result to both sides of [12, (3.17)] and subsequently using part of the argument in $[\mathbf{1 2}$, p. 513] establishes the validity of (1.2).

Summarizing we see that there is a one-to-one correspondence between birth-death processes which are not uniquely determined by their rates on the one hand, and pairs $\{R, \gamma\}$, where $R$ is a rate set defining an IRP and $\gamma$ a real number in the interval indicated in Theorem 2 on the other.

It is also interesting to observe that there is a one-to-one correspondence between birth-death processes which are not uniquely determined by their rates on the one hand, and pairs $\{\mu, d \psi\}$ on the other, where $\mu \geq 0$ and $d \psi$ is an extremal solution of an indeterminate SMP with total mass 1 such that

$$
\mu \int_{0}^{\infty} x^{-1} d \psi(x) \leq 1
$$


The parameter $\mu$ should of course be identified with the death rate in state 0 , and $d \psi$ with the measure in the representation formula (4.1) of the pertinent birth-death process. Conversely, it may be shown that the condition (4.6) ensures that polynomials $Q_{n}$ (and hence rates $\lambda_{l}$ and $\mu_{i}$ ) exist, which are of the form (2.1) with $\mu_{0}=\mu$, and which satisfy (2.10).

We finally mention the references [1], [5], [22] and [25] in relation to the problem of determining, either exactly or approximately, $\xi_{1}(R)$ from the corresponding rate set $R$.

5. Honesty, minimal and maximal processes. In this section we will investigate whether a birth-death process represented by a pair $\{R, \gamma\}$, where $R$ is a rate set defining an IRP and $\gamma$ is a real number in the range allowed by Theorem 2, is honest or not. Here honesty means that the corresponding transition probabilities are such that equality prevails in (1.2) for all $t \geq 0$ and $i \in E^{\prime}$. Note that the prevalence of equality in (1.2) for all $t \geq 0$ is evident if $i=-1$, so we can confine attention to the case $i \in E$. We should remark that some authors call a process honest if and only if

$$
\sum_{j=0}^{\infty} p_{i j}(t)=1
$$

( $i \in E, t \geq 0$ ). In our opinion it is more natural to ask for conditions ensuring equality in (1.2), where the sum includes the probability $p_{i,-1}(t)$. Moreover, this definition of honesty makes the problem analytically more tractable. Actually, the fact that Karlin and McGregor's [12] analysis of the case $\mu_{0}>0$ is less complete than that of the case $\mu_{0}=0$, is partly due to their disregarding the probability $p_{i,-1}(t)$.

As before we write $p_{i j}(t ; \gamma)$ for the transition probabilities of the birth-death process with rate set $R$ and decay parameter $\gamma$. From [12, Thm. 13] we then have

$$
\gamma_{1}<\gamma_{2} \Rightarrow p_{i j}\left(t ; \gamma_{1}\right) \geq p_{i j}\left(t ; \gamma_{2}\right)
$$

( $t \geq 0, i, j \in E$ ). Considering (4.2), an analogous result is valid if $j=-1$. Thus, in view of Theorem 2, we are justified in calling the process with rate set $R$ and decay parameter $\xi_{1}(R)$ the minimal process corresponding to $R$, and the process with rate set $R$ and decay parameter 0 (if $\mu_{0}=0$ ) or $\xi_{1}\left(R^{d}\right)$ (if $\mu_{0}>0$ ) the maximal process corresponding to $R$. Indeed, our minimal process is of course the minimal process in the sense of Feller [10], [11], [17]. Karlin and McGregor's [12] concept of maximal process, however, differs from the one given here, at least if $\mu_{0}>0$. 
Considering the preceding and Reuter's [16] result that there is precisely one honest process with rate set $R$ (if the latter defines an IRP), it follows immediately that the maximal process corresponding to $R$ is the only honest process with rate set $R$.

We note that this result can also be obtained more directly from Lemma 2, (3.10)-(3.12) and the relations

$$
\sum_{j \in E^{\prime}} p_{i j}(t ; \gamma)=\int_{0}^{\infty} e^{-x t} Q_{i}(x) Q_{\infty}^{d}(x) d \psi(x, \gamma)
$$

if $\mu_{0}=0$, and

$$
\begin{aligned}
\sum_{j \in E^{\prime}} p_{i j}(t ; \gamma)= & 1-Q_{i}(0)\left\{1-\mu_{0} \int_{0}^{\infty} x^{-1} d \psi(x ; \gamma)\right\} \\
& -\mu_{0} \int_{0}^{\infty} e^{-x t} x^{-1} Q_{i}(x) Q_{\infty}^{d}(x) d \psi(x ; \gamma)
\end{aligned}
$$

if $\mu_{0}>0$, the proofs of which may be based on results in [12].

The condition that $R$ defines an IRP is in Feller's [11] terminology equivalent to infinity being a regular boundary. The maximal process then corresponds to the solution with a regular reflecting boundary, while the minimal process is the one with a regular absorbing boundary; otherwise the regular boundary is of mixed type [3]. From a probabilistic point of view the maximal process might be called the "natural" solution of the IRP associated with $R$, since it is honest. On the other hand, from an analytical point of view the minimal process is the "natural" solution, because of Feller's construction [10], [11], but also because the measure associated with the minimal solution is, in Chihara's terminology [6], [7], the "best" solution of the indeterminate SMP associated with $R$.

6. Duality for birth-death processes. The duality concept for rate sets introduced in $\$ 3$ implies a duality concept for birth-death processes if the pertinent rate sets determine the corresponding processes uniquely. This duality concept for processes is equivalent to that introduced by Siegmund [19] (see also [8] and [9]) in a more general context. Namely, we have [19] (cf. also [21])

$$
\sum_{j>k} p_{i j}(t)=\sum_{j<i} p_{k j}^{d}(t)
$$

for all $i, k \in E^{\prime}$ and $t \geq 0$, if $\left\{p_{i j}(t)\right\}$ represents a birth-death process which is uniquely determined by its rate set $R \equiv\left\{\lambda_{i}, \mu_{l}\right\}$, where $\mu_{0}=0$, 
and $\left\{p_{i j}^{d}(t)\right\}$ represents the process determined by $R^{d}$. Here $\sum_{j>k} p_{i j}(t)$ should be interpreted as $1-\sum_{j \leq k} p_{i j}(t)$; in other words, if the process represented by $\left\{p_{i j}(t)\right\}$ is not honest, then the disappearing probability mass is assigned to a state $\infty$.

Now let us assume that the rate set $R$, where $\mu_{0}=0$, defines an IRP, so that by virtue of (3.3) there is also an IRP associated with $R^{d}$. As usual we denote by $p_{i j}(t ; \gamma)\left(p_{i j}^{d}(t ; \gamma)\right)$ the transition probabilities of the birth-death process with rate set $R\left(R^{d}\right)$ and decay parameter $\gamma$. The question then arises of whether we can extend the duality concept for rate sets to a duality concept for pairs $\{R, \gamma\}$ such that the corresponding processes satisfy the relation (6.1).

Let us first consider a case for which Siegmund himself gives the solution. Namely, the process $\left\{p_{t j}\left(t ; \xi_{1}(R)\right)\right\}$ is dual in the sense of (6.1) with respect to an honest process with rate set $R^{d}$. Since there is only one honest process with rate set $R^{d}$, viz., $\left\{p_{i j}^{d}\left(t ; \xi_{1}(R)\right)\right\}$, we conclude that $\left\{R, \xi_{1}(R)\right\}$ and $\left\{R^{d}, \xi_{1}(R)\right\}$ are dual pairs in the sense that the corresponding processes satisfy (6.1). In view of Lemma 2 we also know the relation between the measures associated with these dual pairs.

The second case we consider is the process $\left\{p_{i j}(t ; 0)\right\}$ or equivalently, the pair $\{R, 0\}$. It is not surprising that this pair is dual to the pair $\left\{R^{d}, \xi_{1}\left(R^{d}\right)\right\}$ in the sense that the corresponding processes satisfy (6.1). The proof follows from Theorem 2 and will be omitted.

Thus we see that the duality concept for rate sets can be extended to pairs $\{R, \gamma\}$, while maintaining validity of (6.1), if one restricts oneself to minimal and maximal processes: the minimal (maximal) process corresponding to $R$ is dual to the maximal (minimal) process corresponding to $R^{d}$.

Finally consider a pair $\{R, \gamma\}$, where $0<\gamma<\xi_{1}(R)$. We then have in particular

$$
\sum_{j>0} p_{0 j}(t ; \gamma)=1-p_{00}(t ; \gamma)=1-\int_{0}^{\infty} e^{-x t} d \psi(x ; \gamma)
$$

If for the pair $\{R, \gamma\}$ there exists a dual pair $\left\{R^{d}, \xi\right\}$ such that the corresponding processes satisfy (6.1), then, by (4.3), we must have

$$
\begin{aligned}
\sum_{j>0} p_{0 j}(t ; \gamma) & =\sum_{j<0} p_{0 j}^{d}(t ; \xi)=p_{0,-1}^{d}(t ; \xi) \\
& =\mu_{0}^{d} \int_{0}^{\infty} x^{-1} d \psi^{d}(x ; \xi)-\mu_{0}^{d} \int_{0}^{\infty} x^{-1} e^{-x t} d \psi^{d}(x ; \xi)
\end{aligned}
$$


Comparing (6.2) and (6.3) for $t \rightarrow \infty$, we see from Lemma 1 and (3.10) that the only possibility is $\xi=\xi_{1}(R)$, but then, as we have seen, $\gamma=\xi_{1}(R)$, which is a contradiction. So beyond minimal and maximal processes, an extension of the duality concept is not possible.

\section{REFERENCES}

[1] G. Bordes and B. Roehner, Application of Stieltjes theory for S-fractions to birth and death processes, Adv. in Appl. Probab., 15 (1983), 507-530.

[2] H. Callaert, On the rate of convergence in birth-and-death processes, Bull. Soc. Math. Belg., 26 (1974), 173-184.

[3] H. Callaert and J. Keilson, On exponential ergodicity and spectral structure for birth-death processes I, Stochastic Process. Appl., 1 (1973), 187-216.

[4] T. S. Chihara, Convergent sequences of orthogonal polynomials, J. Math. Anal. Appl., 38 (1972), 335-342.

[5] _ An Introduction to Orthogonal Polynomials, (1978) Gordon and Breach, New York.

[6] , Indeterminate symmetric moment problems, J. Math. Anal. Appl., 85 (1982), $331-346$.

[7] Orthogonal polynomials with discrete spectra on the real line, J. Approx. Theory, 42 (1984), 97-105.

[8] P. Clifford and A. Sudbury, A sample path proof of the duality for stochastically monotone Markov processes, Ann. Probab., 13 (1985), 558-565.

[9] J. T. Cox and U. Rösler, A duality relation for entrance and exit laws for Markov processes, Stochastic Process. Appl., 16 (1983), 141-156.

[10] W. Feller, On the integro-differential equations of purely discontinuous Markoff processes, Trans. Amer. Math. Soc., 48 (1940), 488-515; and Errata, 58 (1945), 474.

[11] _ The birth and death processes as diffusion processes, J. Math. Pures. Appl., 38 (1959), 301-345.

[12] S. Karlin and J. L. McGregor, The differential equations of birth-and-death processes, and the Stieltjes moment problem, Trans. Amer. Math. Soc., 85 (1957), 489-546.

[13] S. Karlin and J. L. McGregor, The classification of birth and death processes, Trans. Amer. Math. Soc., 86 (1957), 366-400.

[14] J. H. B. Kemperman, An analytical approach to the differential equations of the birth-and-death process, Michigan Math. J., 9 (1962), 321-361.

[15] J. F. C. Kingman, The exponential decay of Markov transition probabilities, Proc. London Math. Soc., 13 (1963), 337-358.

[16] G. E. H. Reuter, Denumerable Markov processes and the associated contraction semigroups on l, Acta Math., 97 (1957), 1-46.

[17] G. E. H. Reuter and W. Ledermann, On the differential equations for the transition probabilities of Markov processes with enumerably many states, Proc. Cambridge Philos. Soc., 49 (1953), 247-262.

[18] J. Shohat and J. D. Tamarkin, The Problem of Moments, Math. Surveys, No. 1, Amer. Math. Soc., (1943, 1950), Providence, R. I.

[19] D. Siegmund, The equivalence of absorbing and reflecting barrier problems for stochastically monotone Markov processes, Ann. Probab., 4 (1976), 914-924.

[20] T. J. Stieltjes, Recherches sur les fractions continues, Oeuvres, Tome II, P. Noordhoff, Groningen. (1918), 398-566. 
[21] E. A. Van Doorn, Stochastic monotonicity of birth-death processes, Adv. in Appl. Probab. 12 (1980), 59-80.

[22] _ On oscillation properties and the interval of orthogonality of orthogonal polynomials, SIAM J. Math. Anal., 15 (1984), 1031-1042.

[23] _ Conditions for exponential ergodicity and bounds for the decay parameter of a birth-death process, Adv. in Appl. Probab., 17 (1985), 514-530.

[24] _ On orthogonal polynomials with positive zeros and the associated kernel polynomials, J. Math. Anal. Appl., 113 (1986), 441-450.

[25] Representations and bounds for zeros of orthogonal polynomials and eigenvalues of sign-symmetric tri-diagonal matrices, J. Approx. Theory, (1987).

Received August 5, 1986.

UNIVERSITY OF TWENTE

P. O. Box 217

7500 AE ENSCHEDE

THE NETHERLANDS 



\section{EDITORS}

\author{
V. S. VARADARAJAN \\ (Managing Editor) \\ University of California \\ Los Angeles, CA 90024 \\ HeRbert Clemens \\ University of Utah \\ Salt Lake City, UT 84112 \\ R. FINN \\ Stanford University \\ Stanford, CA 94305
}

\author{
HERMANN FLASCHKA \\ University of Arizona \\ Tucson, AZ 85721 \\ RAMESh A. GANGOLLI \\ University of Washington \\ Seattle, WA 98195 \\ VAUGHAN F. R. JONES \\ University of California \\ Berkeley, CA 94720
}

\author{
ROBION KIRBY \\ University of California \\ Berkeley, CA 94720 \\ C. C. MOORE \\ University of California \\ Berkeley, CA 94720 \\ HAROLD STARK \\ University of California, San Diego \\ La Jolla, CA 92093
}

\section{ASSOCIATE EDITORS}
R. ARENS
E. F. BECKENBACH
B. H. NEUMANN
F. WOLF
K. YOSHIDA
(1906-1982)

\section{SUPPORTING INSTITUTIONS}

UNIVERSITY OF ARIZONA
UNIVERSITY OF BRITISH COLUMBIA
CALIFORNIA INSTITUTE OF TECHNOLOGY
UNIVERSITY OF CALIFORNIA
MONTANA STATE UNIVERSITY
UNIVERSITY OF NEVADA, RENO
NEW MEXICO STATE UNIVERSITY
OREGON STATE UNIVERSITY

\author{
UNIVERSITY OF OREGON \\ UNIVERSITY OF SOUTHERN CALIFORNIA \\ STANFORD UNIVERSITY \\ UNIVERSITY OF HAWAII \\ UNIVERSITY OF TOKYO \\ UNIVERSITY OF UTAH \\ WASHINGTON STATE UNIVERSITY \\ UNIVERSITY OF WASHINGTON
}

The Supporting Institutions listed above contribute to the cost of publication of this Journal, but they are not owners or publishers and have no responsibility for its content or policies.

Mathematical papers intended for publication in the Pacific Journal of Mathematics should be in typed form or offset-reproduced (not dittoed), double spaced with large margins. Please do not use built up fractions in the text of the manuscript. However, you may use them in the displayed equations. Underline Greek letters in red, German in green, and script in blue. The first paragraph must be capable of being used separately as a synopsis of the entire paper. In particular it should contain no bibliographic references. Please propose a heading for the odd numbered pages of less than 35 characters. Manuscripts, in triplicate, may be sent to any one of the editors. Please classify according to the scheme of Math. Reviews, Index to Vol. 39. Supply name and address of author to whom proofs should be sent. All other communications should be addressed to the managing editor, or Elaine Barth, University of California, Los Angeles, California 90024.

There are page-charges associated with articles appearing in the Pacific Journal of Mathematics. These charges are expected to be paid by the author's University, Government Agency or Company. If the author or authors do not have access to such Institutional support these charges are waived. Single authors will receive 50 free reprints; joint authors will receive a total of 100 free reprints. Additional copies may be obtained at cost in multiples of 50 .

The Pacific Journal of Mathematics is issued monthly as of January 1966. Regular subscription rate: $\$ 190.00$ a year (5 Vols., 10 issues). Special rate: $\$ 95.00$ a year to individual members of supporting institutions.

Subscriptions, orders for numbers issued in the last three calendar years, and changes of address should be sent to Pacific Journal of Mathematics, P.O. Box 969, Carmel Valley, CA 93924, U.S.A. Old back numbers obtainable from Kraus Periodicals Co., Route 100, Millwood, NY 10546.

The Pacific Journal of Mathematics at P.O. Box 969, Carmel Valley, CA 93924 (ISSN 0030-8730) publishes 5 volumes per year. Application to mail at Second-class postage rates is pending at Carmel Valley, California, and additional mailing offices. Postmaster: send address changes to Pacific Journal of Mathematics, P.O. Box 969, Carmel Valley, CA 93924.

PUBLISHED BY PACIFIC JOURNAL OF MATHEMATICS, A NON-PROFIT CORPORATION Copyright (c) 1987 by Pacific Journal of Mathematics 


\section{Pacific Journal of Mathematics}

\section{Vol. 130, No. $2 \quad$ October, 1987}

Bernhard Banaschewski, J. L. Frith and C. R. A. Gilmour, On the congruence lattice of a frame ............................ 209

Paul S. Bourdon, Density of the polynomials in Bergman spaces ........2215

Lawrence Jay Corwin, Approximation of prime elements in division

algebras over local fields and unitary representations of the multiplicative group

Stephen R. Doty and John Brendan Sullivan, On the geometry of extensions of irreducible modules for simple algebraic groups

Karl Heinz Dovermann and Reinhard Schultz, Surgery of involutions

with middle-dimensional fixed point set ....................2 275

Ian Graham, Intrinsic measures and holomorphic retracts ............ 299

John Robert Greene, Lagrange inversion over finite fields . . . . . . . . . . . 313

Kristina Dale Hansen, Restriction to $\mathrm{GL}_{2}(\mathrm{O})$ of supercuspidal

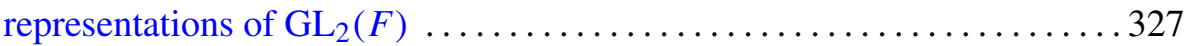

Kei Ji Izuchi, Unitary equivalence of invariant subspaces in the polydisk ....351

A. Papadopoulos and R. C. Penner, A characterization of pseudo-Anosov

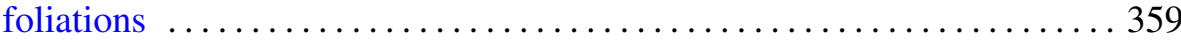

Erik A. van Doorn, The indeterminate rate problem for birth-death

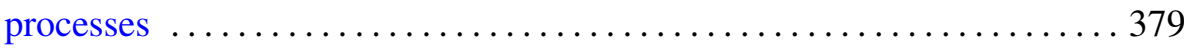

Ralph Jay De Laubenfels, Correction to: "Well-behaved derivations on

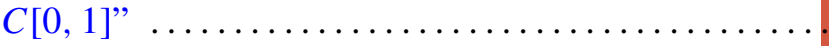

Robert P. Kaufman, Correction to: "Plane curves and removable sets" . . . . 396

Richard Scott Pierce and Charles Irvin Vinsonhaler, Correction to:

"Realizing central division algebras" 